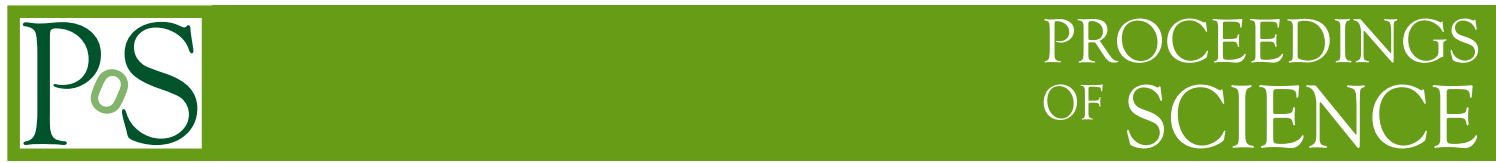

\title{
Progress in the quest for a realistic three-nucleon force
}

\section{Luca Girlanda*}

Department of Mathematics and Physics, University of Salento, and INFN Lecce, Italy

E-mail: luca.girlanda@le.infn.it

\section{Alejandro Kievsky}

INFN Pisa, Italy

E-mail: alejandro.kievskyepi.infn.it

\section{Michele Viviani}

INFN Pisa, Italy

E-mail: michele.viviani@pi.infn.it

\section{Laura Elisa Marcucci}

Department of Physics, University of Pisa, and INFN Pisa, Italy

E-mail: laura.elisa.marcucci@unipi.it

\begin{abstract}
We investigate whether the subleading three nucleon contact interaction, depending on 10 lowenergy constants (LECs), may provide enough flexibility to solve existing discrepancies between theory and experiment in $A=3$ scattering observables. To this aim we choose a realistic twonucleon interaction (AV18) supplemented with the leading and subleading three-nucleon contact interaction, and fit the LECs to very precise data on polarization observables in elastic $p-d$ scattering at $3 \mathrm{MeV}$ proton energy.
\end{abstract}

The 8th International Workshop on Chiral Dynamics, CD2015 ***

29 June 2015 - 03 July 2015

Pisa,Italy

\footnotetext{
* Speaker.
} 


\section{Introduction}

The determination of an accurate nuclear interaction represents a central goal of research in nuclear physics, with important bearings on other fields like astrophysics and particle physics, where a clear assessment of the theoretical uncertainty associated to the nuclear physics input is often crucial. In this respect, modern nuclear interactions based on chiral perturbation theory (ChPT) [1], can be viewed as a definite improvement, since they also allow to estimate the error due to the truncation of the low-energy expansion [2]. In the two-nucleon $(N N)$ sector it seems that the above expansion scheme, pursued at the next-to-next-to-next-to leading order (N3LO) level, is enough to describe the data with high accuracy, with a $\chi^{2} /$ d.o.f. close to one [3]. In the three-nucleon $(3 N)$ sector the situation is far less satisfactory. Indeed, while the ChPT scheme is extremely predictive for the three-nucleon interaction (TNI), in the sense that only two LECs appear up to N3LO [4] (actually only one of them is a truly three-nucleon LEC), preliminary investigations [5] show that strong discrepancies persist at this order between theory and experiment, most notably the so-called $N-d A_{y}$ puzzle, a problem that is shared also by the phenomenological models like e.g. AV18 + IL7 $[6,7,8]$. This could signal a slower convergence of the chiral expansion than observed in the $N N$ sector. The predictive power of the effective theory would then worsen, and higher order LECs should be adjusted in order to accurately describe data. In Ref. [9] we classified all subleading $3 N$ contact terms, compatible with the discrete symmetry of QCD and with the relativity constraints [10], which would contribute at N4LO of the chiral expansion, found that they are strongly constrained by the Pauli principle, and produced the explicit form of the associated $3 \mathrm{~N}$ potential in coordinate space, depending on a short-distance cutoff $\Lambda$ and 10 subleading LECs $E_{i}$, $i=1, \ldots, 10$,

$$
\begin{aligned}
V^{(2)}=\sum_{i \neq j \neq k} & \left(E_{1}+E_{2} \boldsymbol{\tau}_{i} \cdot \boldsymbol{\tau}_{j}+E_{3} \boldsymbol{\sigma}_{i} \cdot \boldsymbol{\sigma}_{j}+E_{4} \boldsymbol{\tau}_{i} \cdot \boldsymbol{\tau}_{j} \boldsymbol{\sigma}_{i} \cdot \boldsymbol{\sigma}_{j}\right)\left[Z_{0}^{\prime \prime}\left(r_{i j}\right)+2 \frac{Z_{0}^{\prime}\left(r_{i j}\right)}{r_{i j}}\right] Z_{0}\left(r_{i k}\right) \\
& +\left(E_{5}+E_{6} \boldsymbol{\tau}_{i} \cdot \boldsymbol{\tau}_{j}\right) S_{i j}\left[Z_{0}^{\prime \prime}\left(r_{i j}\right)-\frac{Z_{0}^{\prime}\left(r_{i j}\right)}{r_{i j}}\right] Z_{0}\left(r_{i k}\right) \\
& +\left(E_{7}+E_{8} \boldsymbol{\tau}_{i} \cdot \boldsymbol{\tau}_{k}\right)(\mathbf{L} \cdot \boldsymbol{S})_{i j} \frac{Z_{0}^{\prime}\left(r_{i j}\right)}{r_{i j}} Z_{0}\left(r_{i k}\right) \\
& +\left(E_{9}+E_{10} \boldsymbol{\tau}_{j} \cdot \boldsymbol{\tau}_{k}\right) \boldsymbol{\sigma}_{j} \cdot \hat{\mathbf{r}}_{i j} \boldsymbol{\sigma}_{k} \cdot \hat{\mathbf{r}}_{i k} Z_{0}^{\prime}\left(r_{i j}\right) Z_{0}^{\prime}\left(r_{i k}\right)
\end{aligned}
$$

where $S_{i j}$ and $(\mathbf{L} \cdot \boldsymbol{S})_{i j}$ are respectively the tensor and spin-orbit operators for particles $i$ and $j$, and the function $Z_{0}(r)$ is the Fourier transform of the cutoff function $F\left(\mathbf{p}^{2} ; \Lambda\right)$,

$$
Z_{0}(r ; \Lambda)=\int \frac{d \mathbf{p}}{(2 \pi)^{3}} \mathrm{e}^{i \mathbf{p} \cdot \mathbf{r}} F\left(\mathbf{p}^{2} ; \Lambda\right) .
$$

Whether the inclusion of these additional terms could lead to an accurate nuclear interaction depends on their flexibility to solve existing discrepancies between theory and experiment for $3 \mathrm{~N}$ observables. It is the purpose of the present paper to investigate this issue. Specifically, we consider a nuclear interaction consisting of the AV18 NN potential [6] and a TNI of the form

$$
V_{3 N}=V^{(0)}+V^{(2)}
$$


where the leading $3 N$ contact potential $V^{(0)}$ is written as

$$
V^{(0)}=\sum_{i \neq j \neq k} E_{0} Z_{0}\left(r_{i j}\right) Z_{0}\left(r_{i k}\right)
$$

We then fit the relevant LECs to the ${ }^{3} \mathrm{H}$ binding energy, the doublet and quarted $N-d$ scattering lengths and accurate scattering data on $p d$ differential cross sections and polarization observables at $3 \mathrm{MeV}$ proton energy [11]. The paper is organized as follows. In Section 2 we describe the variational procedure we use to solve the $p-d$ scattering problem, which is based on the expansion on the Hyperspherical Harmonics (HH method). In section 3 we discuss the isospin properties of the adopted interaction, and restrict ourselves to the isospin $1 / 2$ sector, relevant for the $p-d$ scattering. In section 4 we describe the adopted fitting strategy. Finally, results are reported in section 5 .

\section{Numerical procedure}

The HH method has been reviewed in Ref. [12]. The $N-d$ scattering wave function, below the deuteron breakup threshold, is written as the sum of an internal and an asymptotic part,

$$
\Psi_{L S J J_{z}}=\Psi_{\mathrm{C}}+\Psi_{\mathrm{A}}
$$

where the internal part is expanded on the $\mathrm{HH}$ basis,

$$
\Psi_{C}=\sum_{\mu} c_{\mu} \Phi_{\mu}
$$

$\mu$ denoting a set of quantum numbers necessary to completely specify the basis element, while the asymptotic part describes the relative motion between the nucleon and the deuteron at large separation, which takes the form of a linear combination of the regular and irregular solutions of the free (or Coulomb) $N-d$ Schroedinger equation at relative momentum $q$ (corresponding to energy $E$ ), duly regulated at small distance, $\Omega_{L S J J_{z}}^{\lambda}$ with $\lambda=R, I$ respectively,

$$
\Psi_{A}^{L S J J_{z}}=\Omega_{L S J J_{z}}^{R}+\sum_{L^{\prime} S^{\prime}} \mathscr{R}_{L S, L^{\prime} S^{\prime}}^{J}(q) \Omega_{L^{\prime} S^{\prime} J J_{z}}^{I}
$$

The weights $\mathscr{R}_{L S, L^{\prime} S^{\prime}}^{J}$ of the irregular solution relative to the regular one are related to the $K$ matrix. From them we can determine the scattering phase shifts and mixing parameters, together with the coefficient $c_{\mu}$ in Eq. (2.2), using the Kohn variational principle. The latter requires that the functional

$$
\left[\mathscr{R}_{L S, L^{\prime} S^{\prime}}^{J}(q)\right]=\mathscr{R}_{L S, L^{\prime} S^{\prime}}^{J}(q)-\left\langle\Psi_{L^{\prime} S^{\prime} J J_{z}}|H-E| \Psi_{L S J J_{z}}\right\rangle
$$

be stationary under changes of the variational parameters in $\Psi_{L S J J_{z}}$, with the asymptotic part normalized such that

$$
\left\langle\Omega_{L S J J_{z}}^{R}|H-E| \Omega_{L S J J_{z}}^{I}\right\rangle-\left\langle\Omega_{L S J J_{z}}^{I}|H-E| \Omega_{L S J J_{z}}^{R}\right\rangle=1 .
$$

This implies that weights $\mathscr{R}_{L S, L^{\prime} S^{\prime}}^{J}$ must solve the linear system

$$
\sum_{\tilde{L} \tilde{S}} \mathscr{R}_{L S, \tilde{L} \tilde{S}}^{J} X_{L^{\prime} S^{\prime}, \tilde{L} \tilde{S}}=Y_{L S, L^{\prime} S^{\prime}}
$$


where

$$
X_{L S, L^{\prime} S^{\prime}}=\left\langle\Omega_{L S J J_{z}}^{I}+\Psi_{C}^{I}|H-E| \Omega_{L^{\prime} S^{\prime} J J_{z}}^{I}\right\rangle, \quad Y_{L S, L^{\prime} S^{\prime}}=-\left\langle\Omega_{L S J J_{z}}^{I}+\Psi_{C}^{R}|H-E| \Omega_{L^{\prime} S^{\prime} J J_{z}}^{I}\right\rangle,
$$

and the internal functions $\Psi_{C}^{R / I}$ have coefficients $c_{\mu}^{R / I}$ solutions of

$$
\sum_{\mu^{\prime}} c_{\mu}^{R / I}\left\langle\Phi_{\mu}|H-E| \Phi_{\mu^{\prime}}\right\rangle=-\left\langle\Phi_{\mu}|H-E| \Omega_{L S J J_{z}}^{R / I}\right\rangle .
$$

A second-order estimate is then obtained by substituting the obtained weights $\mathscr{R}_{L S, L^{\prime} S^{\prime}}^{J}$ into Eq. (2.4). Thus the problem is reduced to a linear one: the necessary matrices, e.g. in the 1.h.s. of Eq. (2.8), can be computed as linear combinations of several matrices, one for each operator appearing in $V_{3 N}$, which can be computed once for all, weighted by the corresponding LEC. From the phase shifts we then compute observables by truncating the partial wave expansion to $J \leq 15 / 2$ and $L \leq 6$ : higher partial waves give negligible contributions.

\section{Isospin projection}

Since the deuteron is an isospin singlet, $N-d$ can only give access to the $T=1 / 2$ component of the TNI. We can therefore decompose the contact operators in the two isospin channels, using the projectors

$$
P_{1 / 2}=\frac{1}{2}-\frac{1}{6}\left(\boldsymbol{\tau}_{1} \cdot \tau_{2}+\tau_{1} \cdot \tau_{3}+\tau_{2} \cdot \tau_{3}\right),
$$

and $P_{3 / 2}=1-P_{1 / 2}$. The momentum space potential $V^{(2)}=\sum_{i} E_{i} O_{i}$ involves the 10 operators,

$$
\begin{array}{ll}
O_{1}=-\mathbf{k}_{i}^{2}, & O_{2}=-\mathbf{k}_{i}^{2} \boldsymbol{\tau}_{i} \cdot \boldsymbol{\tau}_{j}, \\
O_{3}=-\mathbf{k}_{i}^{2} \boldsymbol{\sigma}_{i} \cdot \boldsymbol{\sigma}_{j}, & O_{4}=-\mathbf{k}_{i}^{2} \boldsymbol{\sigma}_{i} \cdot \boldsymbol{\sigma}_{j} \boldsymbol{\tau}_{i} \cdot \boldsymbol{\tau}_{j} \\
O_{5}=-3 \mathbf{k}_{i} \cdot \boldsymbol{\sigma}_{i} \mathbf{k}_{i} \cdot \boldsymbol{\sigma}_{j}+\mathbf{k}_{i}^{2} \boldsymbol{\sigma}_{i} \cdot \boldsymbol{\sigma}_{j}, & O_{6}=\left(-3 \mathbf{k}_{i} \cdot \boldsymbol{\sigma}_{i} \mathbf{k}_{i} \cdot \boldsymbol{\sigma}_{j}+\mathbf{k}_{i}^{2} \boldsymbol{\sigma}_{i} \cdot \boldsymbol{\sigma}_{j},\right) \boldsymbol{\tau}_{i} \cdot \tau_{j}, \\
O_{7}=\frac{i}{4} \mathbf{k}_{i} \times\left(\mathbf{Q}_{i}-\mathbf{Q}_{j}\right) \cdot\left(\boldsymbol{\sigma}_{i}+\boldsymbol{\sigma}_{j}\right), & O_{8}=\frac{i}{4} \mathbf{k}_{i} \times\left(\mathbf{Q}_{i}-\mathbf{Q}_{j}\right) \cdot\left(\boldsymbol{\sigma}_{i}+\boldsymbol{\sigma}_{j}\right) \boldsymbol{\tau}_{j} \cdot \boldsymbol{\tau}_{k}, \\
O_{9}=-\mathbf{k}_{i} \cdot \boldsymbol{\sigma}_{i} \mathbf{k}_{j} \cdot \boldsymbol{\sigma}_{j}, & O_{10}=-\mathbf{k}_{i} \cdot \boldsymbol{\sigma}_{i} \mathbf{k}_{j} \cdot \boldsymbol{\sigma}_{j} \boldsymbol{\tau}_{i} \cdot \boldsymbol{\tau}_{j},
\end{array}
$$

with $\mathbf{k}_{i}=\mathbf{p}_{i}-\mathbf{p}_{i}^{\prime}, \mathbf{Q}_{i}=\mathbf{p}_{i}+\mathbf{p}_{i}^{\prime}$ and $\mathbf{p}_{i}\left(\mathbf{p}_{i}^{\prime}\right)$ the initial (final) momentum of the $i$-th nucleon, and a sum over $i \neq j \neq k$ is understood. The projections over isospin $T=1 / 2,\left(O_{i}\right)_{1 / 2}=P_{1 / 2} O_{i} P_{1 / 2}$ are given, using the relations derived in Ref. [9], by

$$
\begin{aligned}
\left(O_{1}\right)_{1 / 2} & =O_{1}-\frac{1}{3} O_{2}+\frac{1}{3} O_{3}+\frac{1}{9} O_{4}+\frac{1}{3} O_{5}+\frac{1}{9} O_{6}-4 O_{7}-\frac{4}{3} O_{8}+O_{9}+\frac{1}{3} O_{10} \\
\left(O_{2}\right)_{1 / 2} & =\frac{2}{3} O_{2}+\frac{1}{3} O_{3}+\frac{1}{9} O_{4}+\frac{1}{3} O_{5}+\frac{1}{9} O_{6}-4 O_{7}-\frac{4}{3} O_{8}+O_{9}+\frac{1}{3} O_{10} \\
\left(O_{i}\right)_{1 / 2} & =O_{i}, \quad i=3, \ldots, 8 \\
\left(O_{9}\right)_{1 / 2} & =\frac{1}{6} O_{2}-\frac{1}{6} O_{3}-\frac{1}{18} O_{4}-\frac{1}{6} O_{5}-\frac{1}{18} O_{6}+2 O_{7}+\frac{2}{3} O_{8}+\frac{1}{2} O_{9}-\frac{1}{6} O_{10} \\
\left(O_{10}\right)_{1 / 2} & =\frac{1}{6} O_{2}-\frac{1}{6} O_{3}-\frac{1}{18} O_{4}-\frac{1}{6} O_{5}-\frac{1}{18} O_{6}+2 O_{7}+\frac{2}{3} O_{8}-\frac{1}{2} O_{9}+\frac{5}{6} O_{10}
\end{aligned}
$$

By examining the above relations, we find that there is only a single purely $T=3 / 2$ combination of operators, e.g.

$$
O_{3 / 2}=3 O_{2}-3 O_{3}-O_{4}-3 O_{5}-O_{6}+36 O_{7}+12 O_{8}-9 O_{9}-3 O_{10} .
$$


Notice that, in order to derive the above projections, Fierz transformations have been heavily used. Therefore the conclusion only holds up to cutoff effects: indeed the cutoff smears the contact interactions, and the three nucleons, which are not anymore at the same positions, are much less constrained by the Pauli principle. As a practical result, we can ignore in our fits one of the operators, e.g. $\mathrm{O}_{2}$.

\section{Fitting strategy}

According to naïve dimensional analysis [13] the expected sizes of the LECs are as follows,

$$
E_{0} \sim \frac{1}{F_{\pi}^{4} \Lambda}, \quad E_{i} \sim \frac{1}{F_{\pi}^{4} \Lambda^{3}}, \quad i=1, \ldots, 10,
$$

where $F_{\pi}$ is the pion decay constant and $\Lambda$ is the hadronic scale. This counting is expected in the pionful theory. In the pionless case the LECs may also receive contributions from virtual pion exchanges, which will produce extra factors of $\Lambda^{2} / M_{\pi}^{2}$. We therefore extract physical dimensions and write

$$
E_{0}=\frac{e_{0}}{F_{\pi}^{4} \Lambda}, \quad E_{i}=\frac{e_{i}}{F_{\pi}^{4} \Lambda^{3}}, \quad i=1, \ldots, 10,
$$

with $e_{0} \sim e_{i} \sim O(1)$ if natural. Also, under the hypothesis of naturalness, there should be a hierarchy, in the sense that $e_{0}$ gives the bulk of the TNI, while the $e_{i}$ should contribute less. Therefore, we first find the LO value of $e_{0}$ by fitting ${ }^{1}$ the triton binding energy $B\left({ }^{3} \mathrm{H}\right)$ and $N-d$ doublet scattering lengths ${ }^{2} a_{N d}$ [15]. This is only possible up to $\sim 10 \%$ theoretical uncertainty, meaning that, in order to achieve a $\chi^{2} /$ d.o.f. $\sim 1$, we have to add a $10 \%$ theoretical uncertainty to the experimental one. We then performed various two-parameters fits of the variables $\left(e_{0}, e_{i}\right)$ to the same observables, for different values of the cutoff $\Lambda$ in the range $200-500 \mathrm{MeV}$, in order to see whether the required $e_{i}$ results to be natural or not. The results are displayed in Table 1. Among the various LECs, $e_{3}$ is the one that allows, when used together with $e_{0}$, to obtain a better description of the two observables, while at the same time respecting the expected naturalness and convergence criteria. We therefore select these particular LECs to account for $B\left({ }^{3} \mathrm{H}\right)$ and ${ }^{2} a_{N d}$. We also show in Fig. 1 fits to six further observables, namely the differential cross section and proton and deuteron polarization observables for $p-d$ scattering at $3 \mathrm{MeV}$ proton energy, to the very precise experimental data of Ref. [11], in the case $\Lambda=300 \mathrm{MeV}$. Not only is the obtained value for $\chi^{2}$ to be considered, but also the actual values of parameters. In particular, we have to prefer minima where $e_{0}$ is not too different with respect to its $\mathrm{LO}$ value. As a general feature, we observe that $T_{20}$ is mostly sensitive to $e_{5}, A_{y}$ and $T_{11}$ to $e_{7}, T_{21}$ to $e_{10}$ and $T_{22}$ to $e_{1}$. This confirms the previously proposed sensitivity of the $A_{y}$ to the spin-orbit interaction [16]. For the differential cross section we included an overall normalization of data points to minimize the $\chi^{2}$, i.e. we defined

$$
\chi^{2}=\sum_{i} \frac{\left(d_{i}^{\mathrm{exp}} / Z-d_{i}^{\mathrm{th}}\right)^{2}}{\left(\sigma_{i} / Z\right)^{2}}
$$

with the normalization $Z$ obtained by minimization,

$$
Z=\frac{\sum_{i} d_{i}^{\exp } d_{i}^{\text {th }} / \sigma_{i}^{2}}{\sum_{i}\left(d_{i}^{\text {th }}\right)^{2} / \sigma_{i}^{2}}
$$

\footnotetext{
${ }^{1}$ All fits were performed using the POUNDerS algorithm [14].
} 


\begin{tabular}{|l|c|c|c|c|}
\hline$\Lambda(\mathrm{MeV})$ & 200 & 300 & 400 & 500 \\
\hline$e_{0} / \chi^{2}$ & $1.27 / 13$ & $0.53 / 40$ & $0.41 / 110$ & $0.45 / 170$ \\
\hline$e_{0} / e_{1}$ & $\mathrm{X}$ & $1.34 /-0.82$ & $1.09 /-0.99$ & $0.89 /-1.45$ \\
$e_{0} / e_{2}$ & $2.38 / 0.84$ & $4.24 / 4.05$ & $1.70 / 2.02$ & $0.90 / 2.02$ \\
$e_{0} / e_{3}$ & $0.39 /-0.95$ & $0.89 / 0.51$ & $0.81 / 0.83$ & $0.65 / 1.27$ \\
$e_{0} / e_{4}$ & $\mathrm{X}$ & $1.45 / 0.33$ & $1.20 / 0.40$ & $0.97 / 0.54$ \\
$e_{0} / e_{5}$ & $1.52 / 1.24$ & $0.15 /-0.90$ & $-0.49 /-1.26$ & $-1.47 /-1.53$ \\
$e_{0} / e_{6}$ & $1.70 /-0.65$ & $-0.03 / 0.38$ & $-0.79 / 0.47$ & $-1.91 / 0.52$ \\
$e_{0} / e_{7}$ & $\mathrm{X}$ & $\mathrm{X}$ & $\mathrm{X}$ & $\mathrm{X}$ \\
$e_{0} / e_{8}$ & $\mathrm{X}$ & $0.93 /-7.95$ & $\mathrm{X}$ & $\mathrm{X}$ \\
$e_{0} / e_{9}$ & $\mathrm{X}$ & $\mathrm{X}$ & $1.37 /-7.29$ & $1.26 /-6.94$ \\
$e_{0} / e_{10}$ & $\mathrm{X}$ & $-0.03 /-4.60$ & $\mathrm{X}$ & $-1.29 /-3.03$ \\
\hline
\end{tabular}

Table 1: Results of 1-parameter (first line) and 2-parameter fits to $B\left({ }^{3} \mathrm{H}\right)$ and ${ }^{2} a_{N d}$ for different values of the cutoff $\Lambda$. A cross indicates that no solution has been found in the natural range.

$\Lambda=300 \mathrm{MeV}$
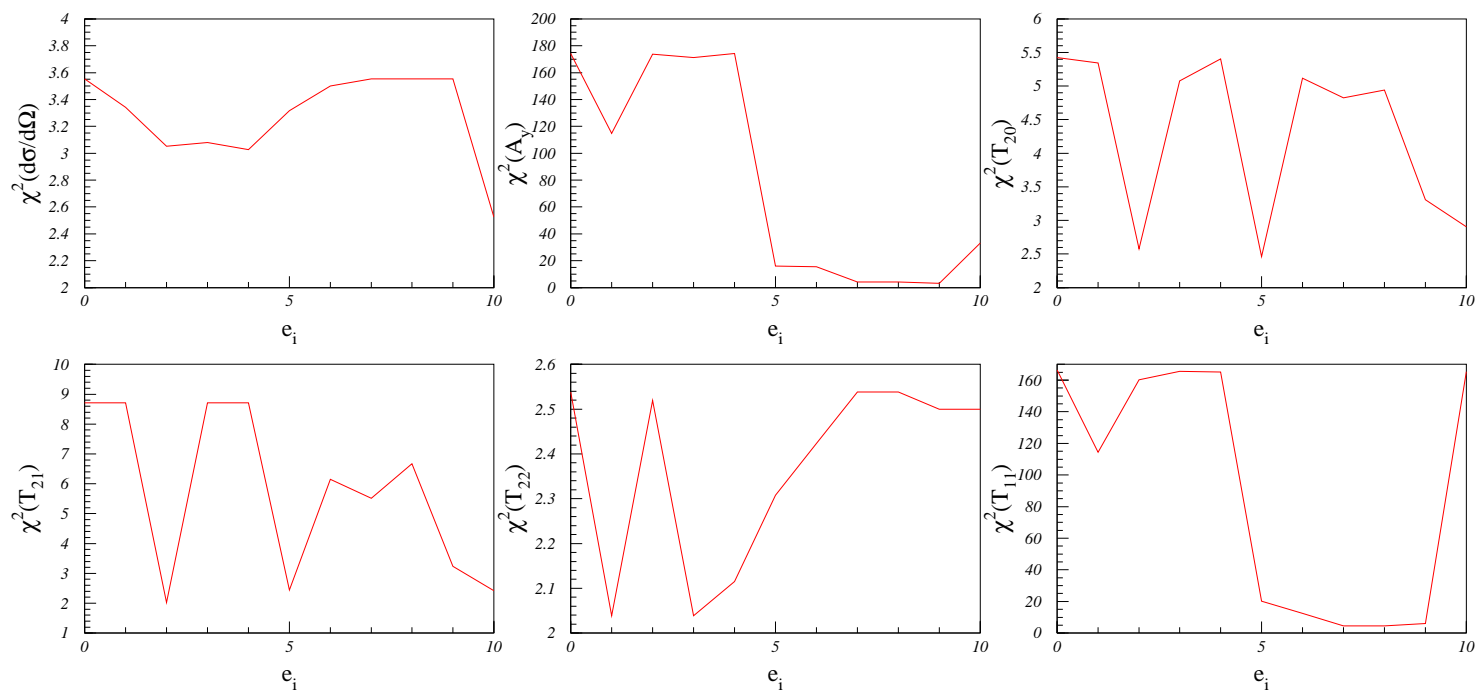

Figure 1: 2-parameter $\left(e_{0}, e_{i}\right)$ fits to six different $p-d$ scattering observables at $3 \mathrm{MeV}$ proton energy, for $\Lambda=300 \mathrm{MeV}$.

and checked that $Z$ never differs from 1 by more than $1 \%$. Actually it turns out that $Z \sim 0.99$ for most of the cases, similarly to the findings of Ref. [17] for the same data. For polarization observables, we took into account the reported systematic uncertainty $\sigma_{\text {sys }}$, i.e. we defined,

$$
\chi^{2}=\sum_{i} \frac{\left(d_{i}^{\exp } / Z-d_{i}^{\text {th }}\right)^{2}}{\left(\sigma_{i} / Z\right)^{2}}+\frac{(Z-1)^{2}}{\sigma_{\text {sys }}^{2}},
$$

and

$$
Z=\frac{\sum_{i} d_{i}^{\exp } d_{i}^{\mathrm{th}} / \sigma_{i}^{2}+\left(1 / \sigma_{\mathrm{sys}}\right)^{2}}{\sum_{i}\left(d_{i}^{\mathrm{th}}\right)^{2} / \sigma_{i}^{2}+\left(1 / \sigma_{\mathrm{sys}}\right)^{2}},
$$


with the overall normalization uncertainty estimated to be within $1 \%$ [11], therefore $\sigma_{\text {sys }}=0.01$. We then proceeded to perform global fits to all observables, also including the $N-d$ quartet scattering length [18], by increasing the number of parameters until a significant reduction in the $\chi^{2} /$ d.o.f. is obtained. For instance, in Fig. 2 we display the results of a 3-parameter fit to all considered observables using the parameters $\left(e_{0}, e_{3}, e_{i}\right), i \neq 3$.

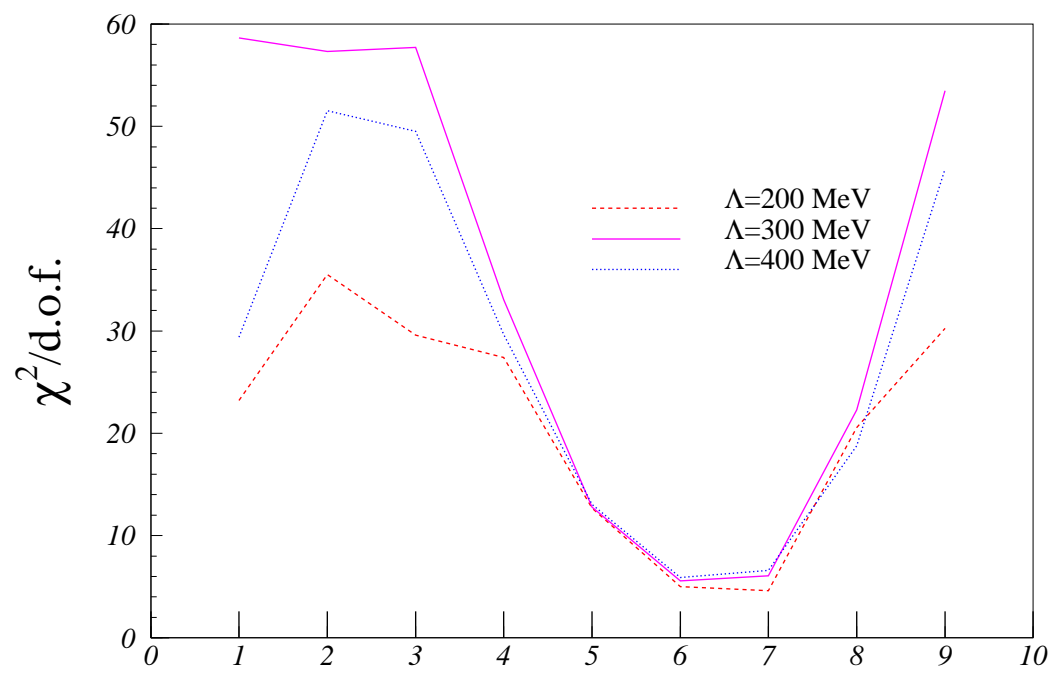

Figure 2: 3-parameter $\left(e_{0}, e_{3}, e_{i}\right)$ fits to $B\left({ }^{3} \mathrm{H}\right),{ }^{2} a_{N d}$ and all considered $p-d$ scattering observables at $3 \mathrm{MeV}$ proton energy, for three different cutoffs $\Lambda$.

\section{Results}

By successively including more parameters we observe a reduction in $\chi^{2}$ down to $\chi^{2} /$ d.o.f. $~$ 1.6. This happens, for $\Lambda=300 \mathrm{MeV}$ when 7, out of the 10 subleading LECs, are used in the fits. This means that there are correlations among the LECs and $p-d$ scattering alone cannot determine all of them. We display in Figs. 3 and 4 the result of a 8-parameter global fit for $\Lambda=300 \mathrm{MeV}$ amd $\Lambda=200 \mathrm{MeV}$. The blue dashed curves are the results corresponding to the AV18 NN interaction without TNI, while the red solid ones are the fit results. As it is apparent, subleading contact interactions allow for a very accurate description of experimental data. In particular, the $A_{y}$ problem is solved, although this observable still gives most of the contribution to the $\chi^{2}$. It should also be remembered that the effective description is the result of truncating a low-energy expansion. Therefore we have to expect a theoretical uncertainty introduced by such truncation. From the fit results we estimate this uncertainty to be of the order of $1 \%$. We should also point out that experimental error are given with only 1 significant digit: a $10 \%$ larger error would further reduce the $\chi^{2} /$ d.o.f. from 1.6 to 1.3 , in line with modern realistic $N N$ potentials in the $N N$ sector [19]. We also show in the figures the results for the triton binding energy and for the $N-d$ scattering lengths. The outcome for the LECs shows that most of them are "natural", i.e. they have a magnitude in line with naïve dimensional analysis. However, we observe, in the case of $\Lambda=200 \mathrm{MeV}$ a change 


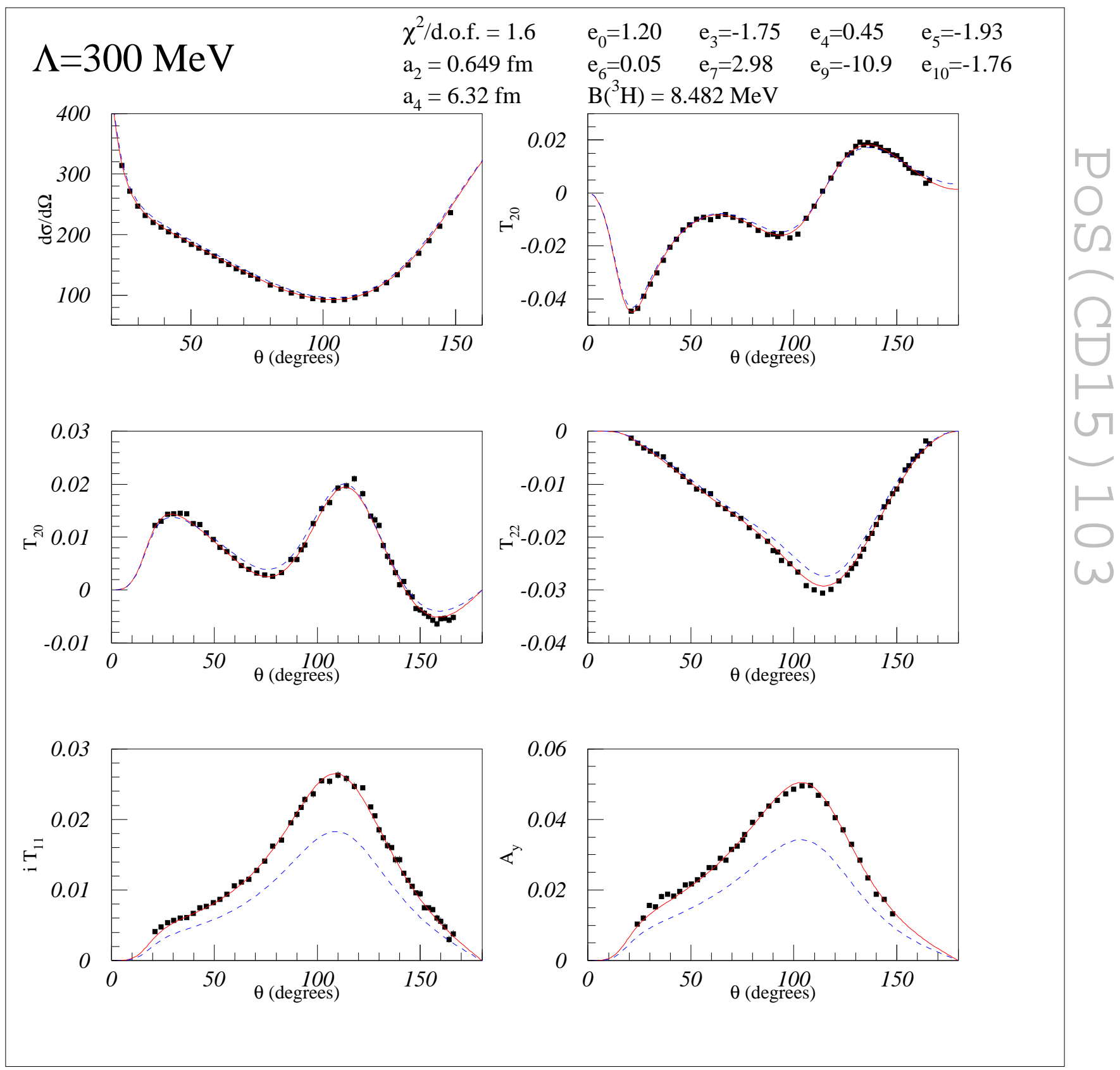

Figure 3: 8-parameter fit to $B\left({ }^{3} \mathrm{H}\right),{ }^{2} a_{N d}$ and all considered $p-d$ scattering observables at $3 \mathrm{MeV}$ proton energy, for $\Lambda=300 \mathrm{MeV}$. Blue dashed curves corresponding to the AV18 NN interaction, while the red solid ones include the fitted TNI. 


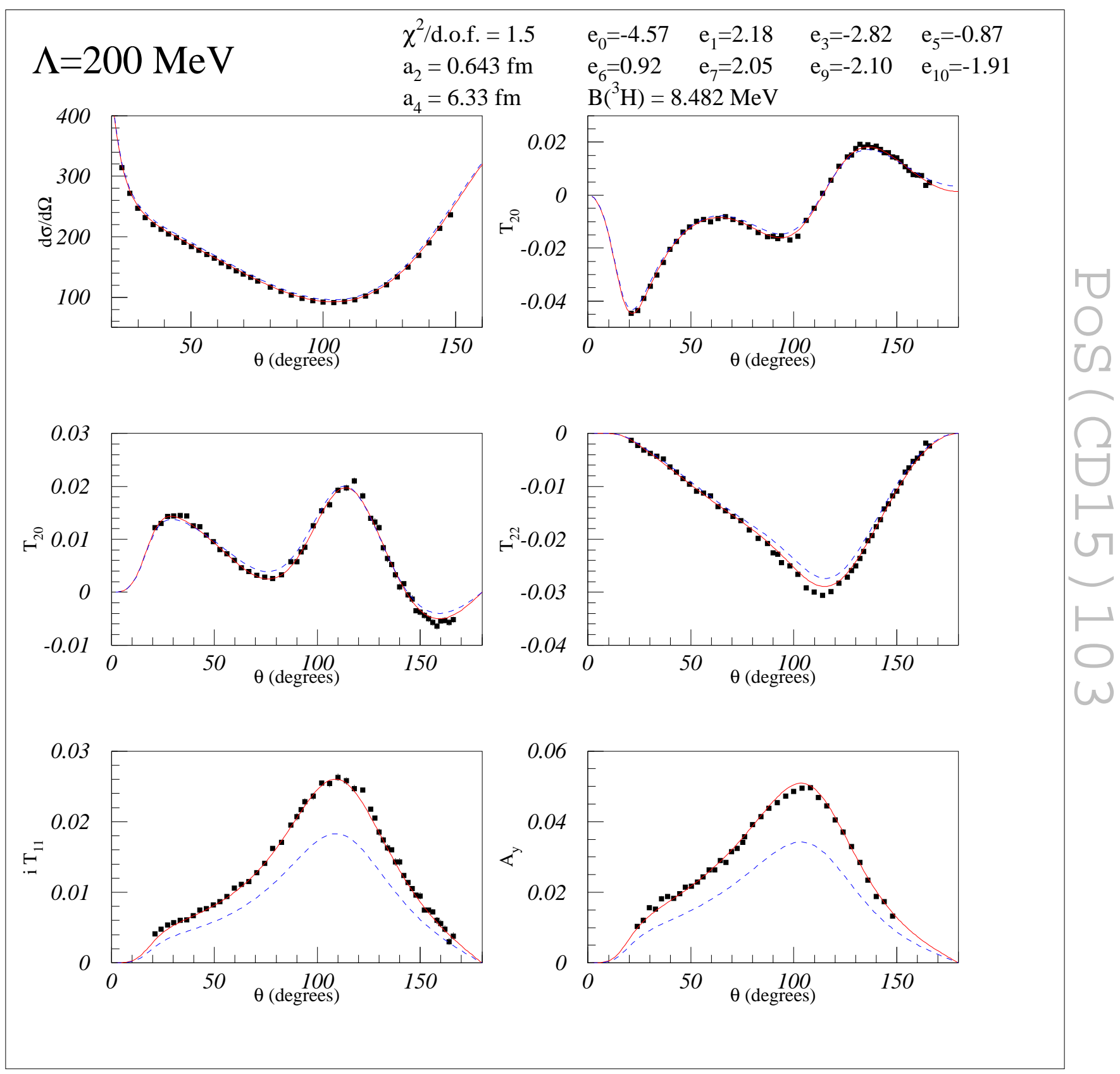

Figure 4: Same as Fig. 3 but for $\Lambda=200 \mathrm{MeV}$.

of sign in the LO LEC, which may depend on the fact that, for such low values of cutoff the convergence of the low-energy expansion is problematic, so that subleading LECs play a nonnegligible role.

Further investigation is necessary in order to explore all the parameter space, to ensure to find a global minimum and to study the cutoff dependence. Studies along these lines are in progress. 


\section{References}

[1] E. Epelbaum, H. W. Hammer and U. G. Meissner, Rev. Mod. Phys. 81, 1773 (2009); R. Machleidt and D. R. Entem, Phys. Rept. 503, 1 (2011).

[2] E. Epelbaum, H. Krebs and U. G. Meissner, Eur. Phys. J. A 51, 53 (2015); Phys. Rev. Lett. 115, 122301 (2015).

[3] D. R. Entem and R. Machleidt, Phys. Rev. C 68, 041001 (2003); E. Epelbaum, W. Glockle and U. G. Meissner, Nucl. Phys. A 747, 362 (2005).

[4] E. Epelbaum, A. Nogga, W. Gloeckle, H. Kamada, U. G. Meissner and H. Witala, Phys. Rev. C 66, 064001 (2002); V. Bernard, E. Epelbaum, H. Krebs and U. G. Meissner, Phys. Rev. C 77, 064004 (2008); Phys. Rev. C 84, 054001 (2011).

[5] J. Golak et al., Eur. Phys. J. A 50177 (2014).

[6] R. B. Wiringa, V. G. J. Stoks and R. Schiavilla, Phys. Rev. C 51, 38 (1995).

[7] S. C. Pieper, V. R. Pandharipande, R. B. Wiringa and J. Carlson, Phys. Rev. C 64, 014001 (2001); S. C. Pieper, AIP Conf. Proc. 1011143 (2008).

[8] M. Viviani, L. Girlanda, A. Kievsky and L. E. Marcucci, Phys. Rev. Lett. 111, 172302 (2013).

[9] L. Girlanda, A. Kievsky and M. Viviani, Phys. Rev. C 84, 014001 (2011).

[10] L. Girlanda, S. Pastore, R. Schiavilla and M. Viviani, Phys. Rev. C 81, 034005 (2010).

[11] S. Shimizu et al., Phys. Rev. C 52, 1193 (1995).

[12] A. Kievsky, S. Rosati, M. Viviani, L. E. Marcucci and L. Girlanda, J. Phys. G 35, 063101 (2008).

[13] A. Manohar and H. Georgi, Nucl. Phys. B 234189 (1984); H. Georgi, Phys. Lett. B 298187 (1993).

[14] T. Munson, J. Sarich, S. Wild, S. Benson and L. McInnes, TAO 2.0 Users Manual, Technical Report ANL/MCS-TM-322, http://www.mcs.anl.gov/tao

[15] K. Schoen et al., Phys. Rev. C 67, 044005 (2003).

[16] A. Kievsky, Phys. Rev. C60 034001 (1999).

[17] A. Kievsky et al., Phys. Rev. C 63024005 (2001).

[18] W. Dilg, L. Koester and W. Nistler, Phys. Lett. B 36208 (1971).

[19] M. Piarulli, L. Girlanda, R. Schiavilla, R. Navarro Pérez, J. E. Amaro and E. Ruiz Arriola, Phys. Rev. C 91, 024003 (2015). 